\title{
The Electric Potential of the Female Body Liquids and the Effectiveness of Cloning
}

\author{
Yuri Pivovarenko* \\ Physical and Chemical Materials Science, Ukraine
}

Received: 眥 February 06, 2018; Published: 眥 February 16, 2018

*Corresponding author: Yuri Pivovarenko, Research and Training Centre 'Physical and Chemical Materials Science' Under Kyiv Taras Shevchenko University and NAS of Ukraine, Kiev, Ukraine

\begin{abstract}
It was previously shown that the electric potential of biological liquids of the female body correlated with the stages of the menstrual cycle: these liquids have a negative potential at the stage of ovulation, but the positive potential on the phases before and after ovulation. It was also shown that the electric potential of the water determines its surface tension, as well as its ability to hydrate the polysaccharides. On the basis of obtained results it is concluded that the electrical potential of the body liquids of women, and is the surface tension and the ability to hydrate the polysaccharides of these liquids, can be cyclically varied during the menstrual cycle. It is demonstrated that the use of these ratios allows influencing the processes of reproduction and increasing the efficiency of cloning.
\end{abstract}

Keywords: Arborisation; Menstrual cycle; Female body liquids; Electric potential; Cloning

\section{Introduction}

It is known that the evaporation of the female body liquids is accompanied by the formation of crystals of different shapes. It is also known that the shape of these crystals depends on the stage of the menstrual cycle: cubic or rhombic crystals are formed before and after the stage of ovulation; arbor-shape crystals are formed in the ovulation step (Figure 1) [1].
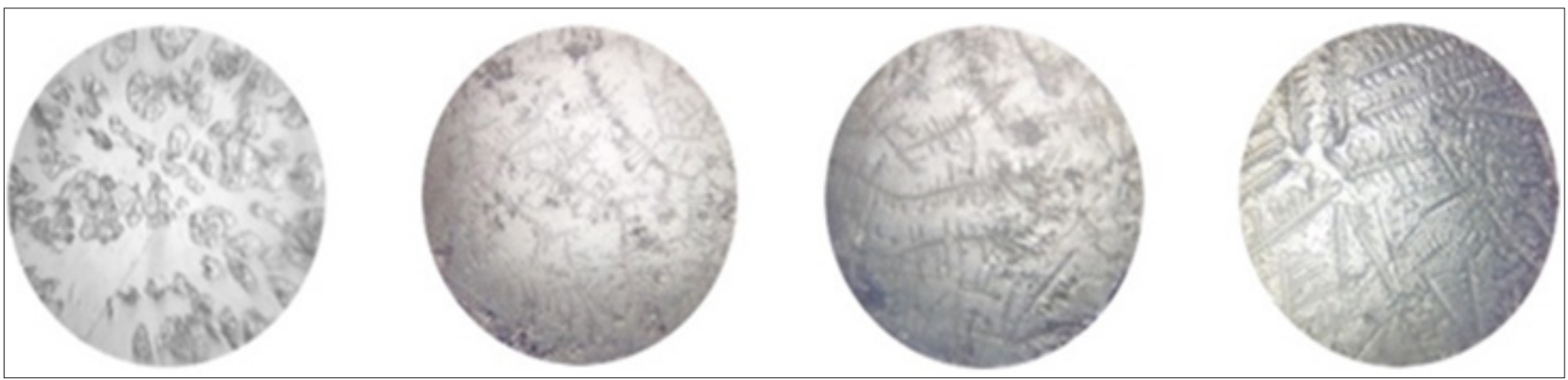

Figure 1: There are crystals which are formed during the evaporation of the liquids of the female body in the first half of the menstrual cycle: from 1 (left) to 14 (right) days [1].

\section{Body}

In the study of the physical nature of this phenomenon, it was discovered that the shape of the crystals formed by evaporation of salt solutions depends on the sign of the electric potential of the solution, and the sign of the electric charge of the surface on which the formation of salt crystals. Cubic or rhombic crystals are formed by evaporation of salt solutions prepared on the water with a positive electric potential (Figure 2, left) or (and) on the positively charged surfaces (Figure 3, left). In contrast, the needle- or arborshaped crystals are formed by evaporation of salt solutions prepared on the water with a negative electric potential (Figure 2, right) or (and) on the negatively charged surfaces (Figure 3, right) [2,3]. It is very important that this correlation is true for $\mathrm{NaCl}$ (Figures $3 \& 4$ ), the main salt component of biological liquids of the human body. 


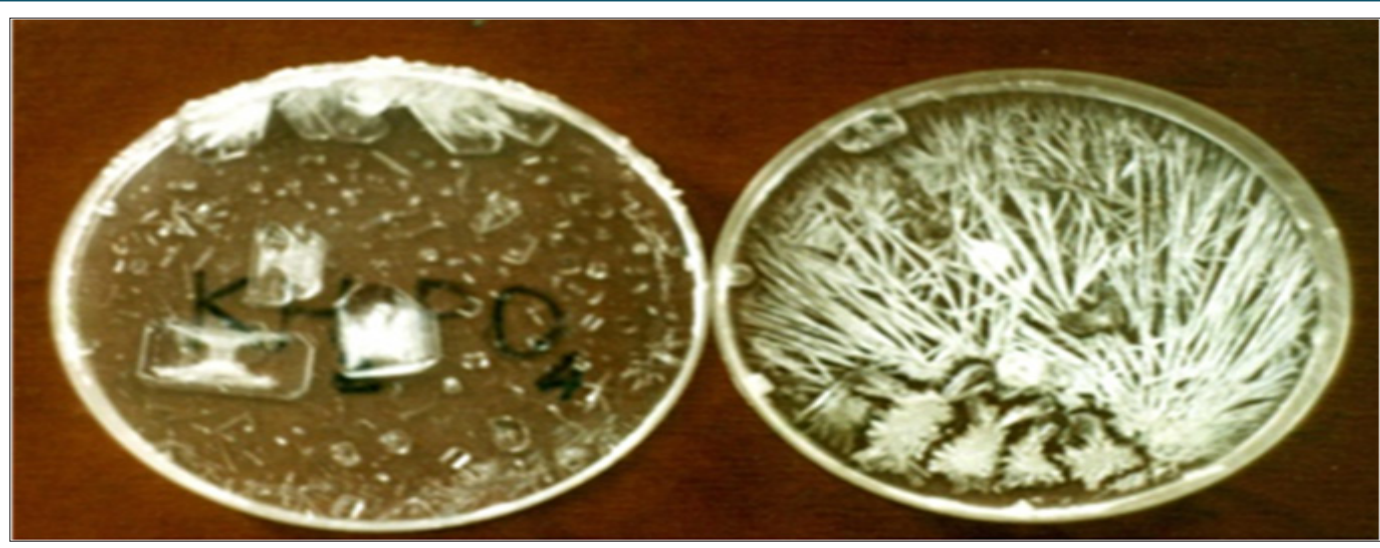

Figure 2: Left: the rhombic crystals formed upon drying of an aqueous solution of $\mathrm{KH} 2 \mathrm{PO} 4$ prepared on the water with positive electric potential. Right: the needle-shaped crystals formed upon drying of an aqueous solution of KH2PO4 prepared on the water with negative electric potential $[2,3]$.

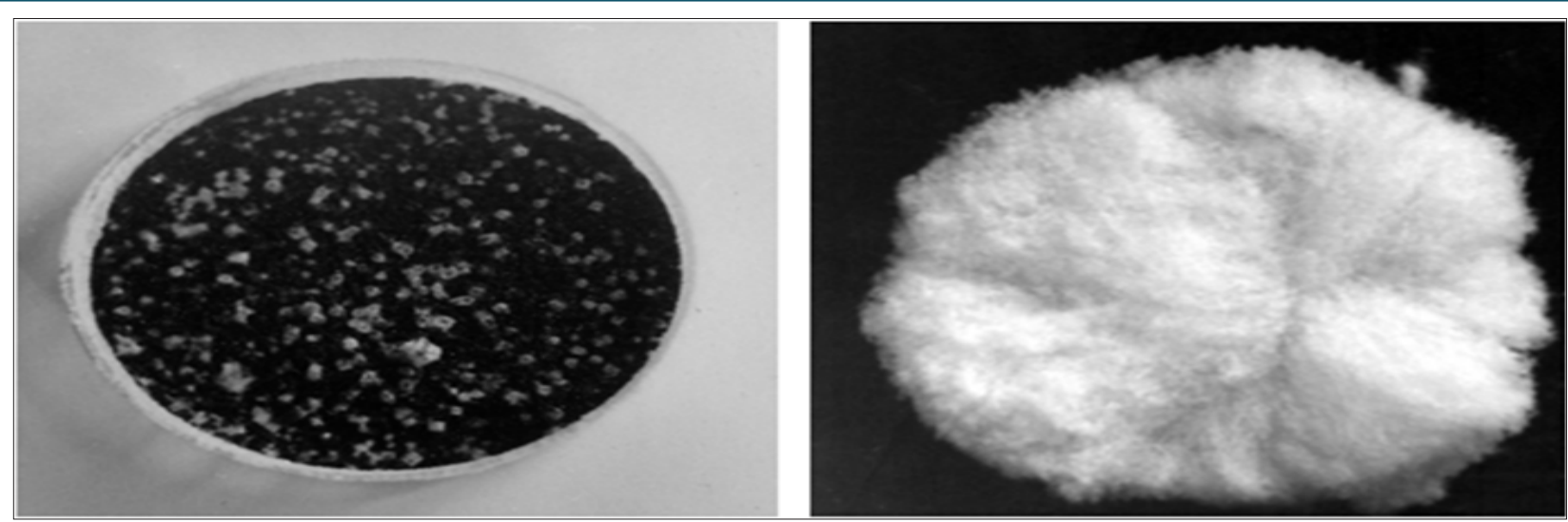

Figure 3: Left: These are the small cubic crystals formed on positively charged surface of activated carbon, pre-wetted with a solution of $\mathrm{NaCl}$. Right: These are the needle-shaped crystals formed on negatively charged surface of silica gel, pre-wetted with a solution of $\mathrm{NaCl}$; in this case, the interlacement of acicular crystals forms a kind of wool [2].

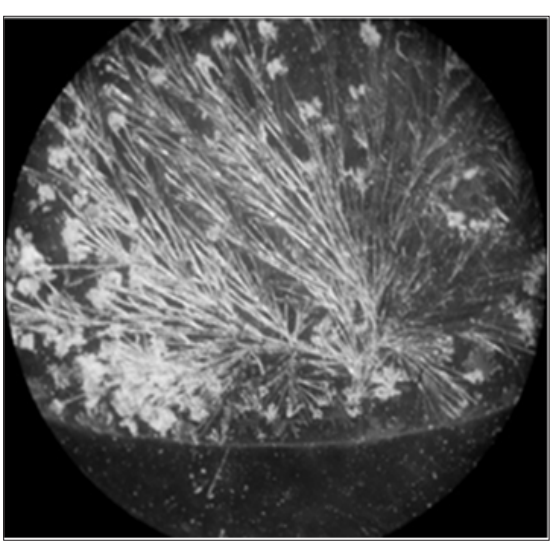

Figure 4: The arbor-shaped crystals formed upon drying of an aqueous solution of $\mathrm{NaCl}$ prepared on the water with negative electric potential [2].

This correlation gave an opportunity to conclude that the various forms of crystals, formed by evaporation of biological liquids of women reflect the changes of electric potential such liquids occurring during the menstrual cycle [2]. In particular, it has been made such findings: a) the menstrual cycle reflects the cyclic changes of the electric potential of biological liquids of the female body;

b) biological liquids of the female body have a positive electric potential (charge) before and after ovulation;

c) the female body liquids have a negative electric potential (charge) in the ovulation step [2].

In further studies, it was found that the surface tension of water also depends on its electric potential. In particular, it was shown that the surface tension of water with a positive potential is always greater than the surface tension of water with negative potential. It is noteworthy that this dependence can be visualized with simple and clear experiments. So, if to pour $5 \mathrm{ml}$ of water (exact!) with a negative potential in a standard Petri dish and mix, it can be saw that the water completely covers the bottom of a Petri dish (Figure 5 , left). On the other hand, if to pour $5 \mathrm{ml}$ of water (exact!) with a positive potential in a standard Petri dish and mix, it can be saw that the water does not cover all the bottom of a Petri dish (Figure 5 , right). 


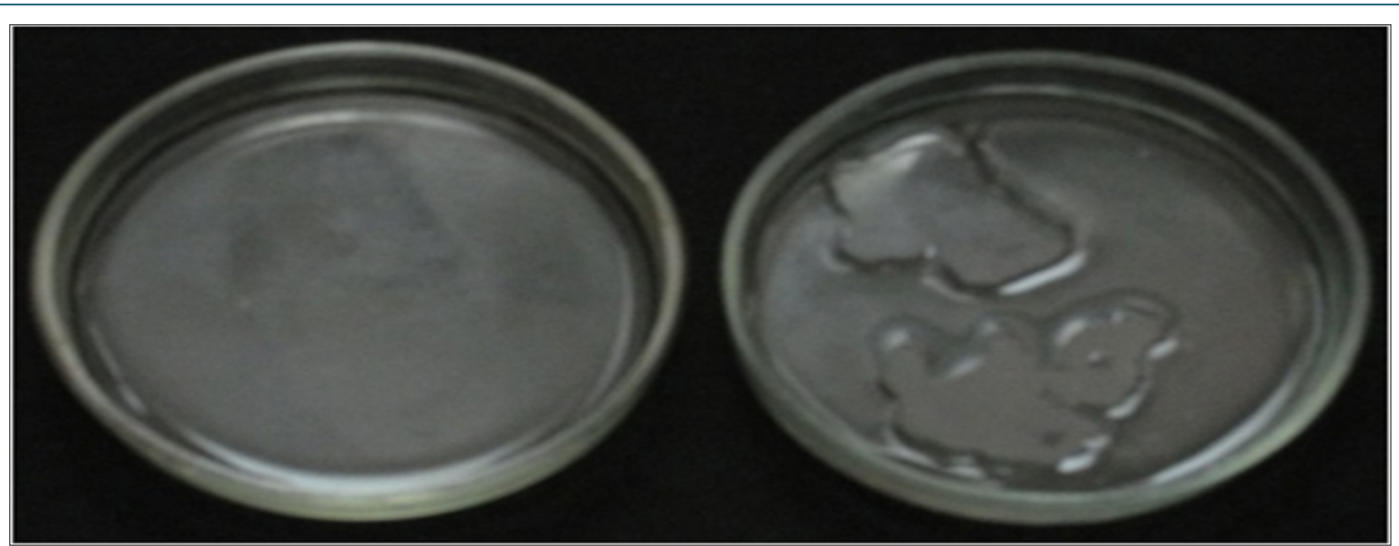

Figure 5: Left: $5 \mathrm{ml}$ of water with an electric potential of $-200 \mathrm{mV}$ cover all the bottom of a Petri dish. Right: $5 \mathrm{ml}$ of water with an electric potential of $+200 \mathrm{mV}$ do not cover the bottom of a Petri dish; the surface of such water decreases rapidly after mixing [3].

The difference can be also visualized by means of a powder of starch deposited on the surface of charged water. By this simple test, it can be saw that the forces acting on the surface of positively charged water dispense powder starch on the surface of the water (Figure 6, left); such a distribution of powder of starch takes place over 1-2 seconds. It can be also said that the surface forces of negatively charged water do not distribute the starch powder across the water surface (Figure 6, right). Moreover, it can be observed that the starch powder sinks into negatively charged water [3]. In our opinion; the experiments clearly demonstrate the difference in the surface tension of water with positive and negative potentials. This can also be concluded that the positive electrisation of the water will increase its surface tension, and the negative electrisation of water will lead to its reduction.
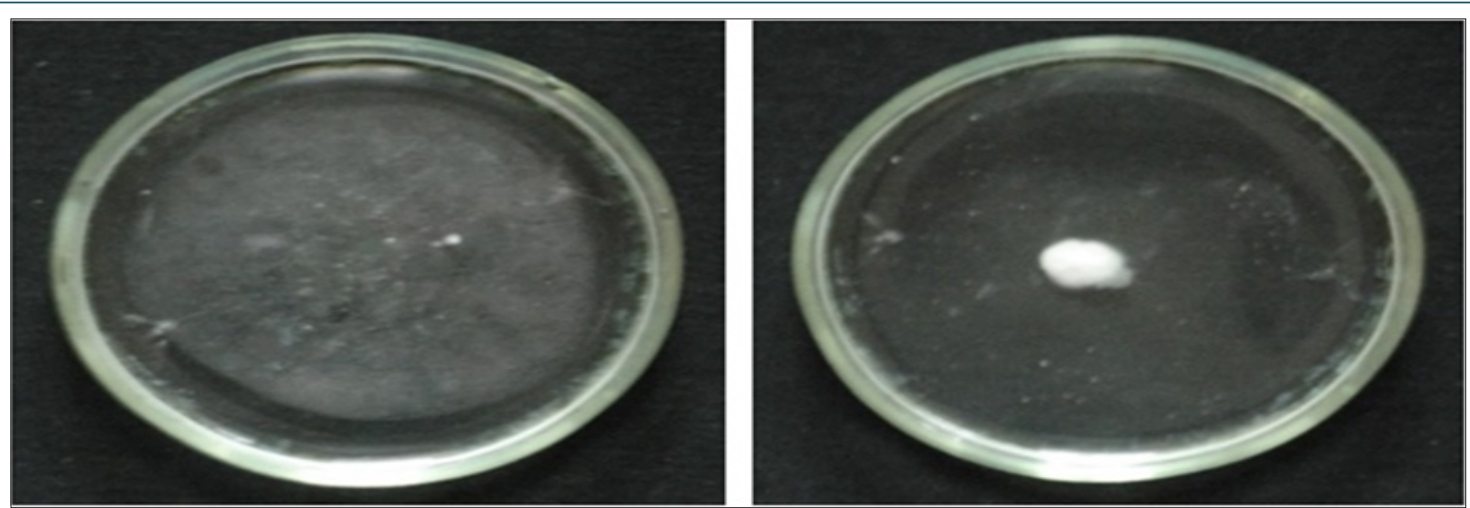

Figure 6: Left: the starch powder covers the surface of the water with potential $+250 \mathrm{mV}$ practically wholly Right: powder starch remains in the same place where it was put in water potential $-200 \mathrm{mV}$ [3].

(It is necessary to say that a similar difference in surface tension is observed also for some other liquids and some solid materials [3]. This provides the fundamental possibility of extrapolation of this dependency to other objects, including biological.) This dependency also helps to explain the main dependence (Figure 2). The high surface tension of water with a positive potential, determines the formation of compact cubic and rhombic crystals. In contrast, the low surface tension of the water with a negative electric potential determines the formation of non-compact needleand arbor-shaped crystals.

On the basis of recent results (Figures $5 \& 6$ ), it is also possible to draw the following assumptions:

a) The surface tension of female body liquids cyclic changes during the menstrual cycle; b) The female body liquids have lowest surface tension in the ovulation step;

c) The female body liquids have the greatest surface tension before and after ovulation.

Extrapolating the basic correlation on the oocytes, it can be assuming that a similar change of the surface tension is also correct with respect to the water that is part of oocyte membranes. Taking this extrapolation, it can be concluded that the reduction of surface tension of the membranes of oocytes occurring at the stage of ovulation, creates optimal conditions for penetration of spermatozoids into oocytes. Using the same extrapolation, it can be also assumed that the increase in surface tension of the membranes of oocytes that occurred before and after ovulation prevents penetration of spermatozoids inside the oocyte. Further studies 
revealed that the electrical potential of the water also determines its ability to hydrate the polymers of biological origin: the water with a positive potential is better hydrates biological polymers than water with negative potential [4]. This dependence it is convenient to show also with starch (Figure 7).

Water with a positive potential can evaporate even from a closed plastic dishes: the arrow shows how during the day decreased the water level to the positive potential. During the last experiment, it was observed another interesting property of water with positive potential. It can be seeing (Figure 7, right), this water is able to penetrate through the plastic and evaporate from a closed polyethylene bottle. (It should be noted that the increase in the permeability of polyethylene to water vapour is known: this phenomenon was observed earlier in the oxidation of polyethylene ROS induced by ionizing radiation [5]. Recently we proposed a detailed analysis of the physical forces causing this phenomenon [6]. Apparently, the high penetrating ability of the water with a positive potential determines the nature of its interaction with polymers, in particular - hydrating ability of this water (Figure 7, right).

Extrapolating the dependence established (Figure 7) on the oocytes, it can be assuming that similar dependence is also correct with respect to polysaccharides that are associated with the outside of the membranes of the oocyte and form a glycocalyx. If this extrapolation is correct, it can be assumed that the decrease of oocyte hydration of the glycocalyx, which occurs at the stage of ovulation, is accompanied by a decrease in the thickness of the glycocalyx, which creates optimal conditions for penetration of spermatozoids into oocytes. Using the same extrapolation, it can also be assumed that the increase in hydration of the glycocalyx of the oocyte that occur before and after ovulation, accompanied by an increase in thickness of the glycocalyx, which prevents the penetration of spermatozoids inside the oocyte.

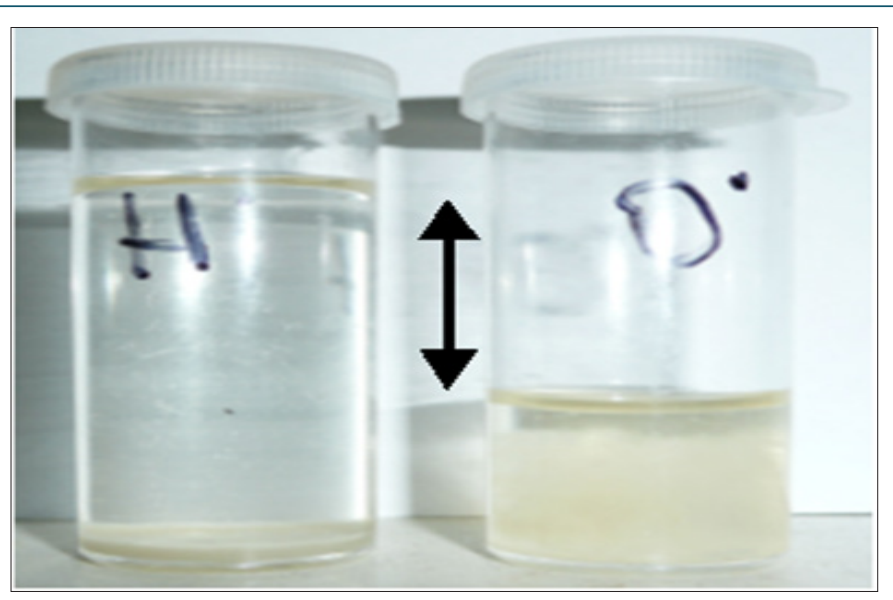

Figure 7: There is a swelling of starch in water with a different electric potential. Starch does not swell in water with the potential of $-500 \mathrm{mV}$ (left) and swells in water with a potential of $+500 \mathrm{mV}$ (right) [3]. Water with negative potential was obtained by bubbling hydrogen (left); water with a positive potential was obtained by bubbling oxygen (right) [4]. The temperature of the water in both vials was $+\left(20-22^{\circ} \mathrm{C}\right)$.

On the basis of last result (Figure 7), it is possible to draw the following assumptions:

a) the menstrual cycle reflects the cyclic change in the thickness of the oocyte glycocalyx;

b) the thickness of the glycocalyx increases in the step preceding ovulation, as well - in the step after ovulation;

c) The thickness of the glycocalyx decreases in the ovulation step.

One of the mostimportantfunctions of glycocalyx connected with its glue ability. (The importance of glycocalyx function is due to the fact that most cells of multi cellular organisms are glued together!) It is obvious that this ability of the glycocalyx depends on the degree of its hydration: hydrated glycocalyx is the glue and dry glycocalyxno. Figure 7 enough adequately displays this dependence. Thus, it can be expected that the adhesive capacity of other polysaccharides is also potential-dependent. If this extrapolation is correct, it can be assumed that a decrease in hydration of the glycocalyx of the oocytes, which occurs at the stage of ovulation, accompanied by a decrease in its adhesive ability, which creates conditions for release of the oocytes from the ovarian follicles. It also suggests that the increase of hydration of the glycocalyx of the oocytes, which occurs after ovulation, can cause a fixation the fertilized egg in the uterus.

Thus, the electric potential of biological liquids of the female body may define two (at least) of a kind of the factors affecting the membrane permeability of the oocytes to spermatozoids. Therefore, there are at least two factors that depend on the electric potential of biological liquids of women that determine the effectiveness of female fertilization. In addition to these factors should also be borne in mind the fact that DNA has an extraordinary sensitivity to the electric potential of the aquatic environment $[7,8]$. In particular, this sensitivity is confirmed by the spectra of UV absorption of DNA solutions prepared in water with a different electric potential 
(Figure 8). Indeed, if the electrical potential of biological fluids affects only the properties of the oocyte membrane, this may turn out to be strange.

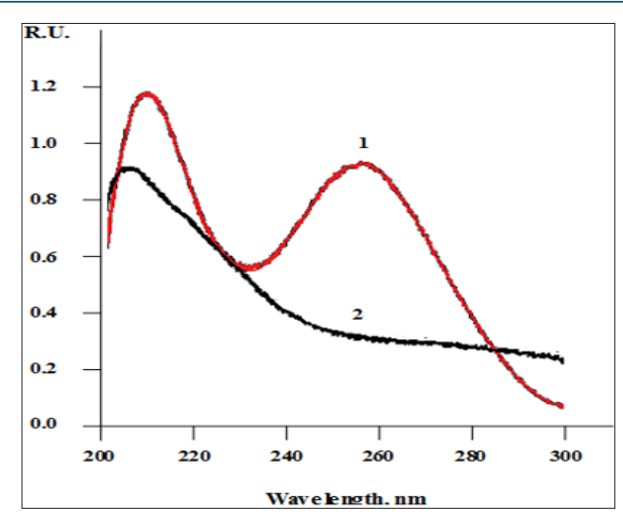

Figure 8: UV absorbance spectra of the aqueous DNA $(\sim 20 \mu \mathrm{g} / \mathrm{ml}): 1$ - DNA, dissolved in the positively charged water; 2 - DNA, dissolved in the positively charged water.

Also, high sensitivity of DNA to the electric potential of the aquatic environment can be demonstrated in an experiment similar to that described above (Figure 7). For these reasons, the electric potential of the tissue liquids of living organisms, it was proposed to accept as an epigenetic factor [8]. In addition, the role of the electrostatic potential of DNA in the organization of the promoter has been demonstrated $[9,10]$. Obviously, the knowledge shown of the dependencies is required for professionals involved in cloning and reproductive technologies, generally. In particular, the demand for such knowledge is dictated by the well-known low efficiency of cloning $[9,10]$. A possible reason for this is the lack of attention to the electric potential of the working solutions (including women's bodily liquids) and hydrated biological objects.

So, usually ignores the fact that the evaporating working solutions and wet objects clearly get a negative charge (potential) due to the evaporation of water from their surfaces [6]. Due to the negative electrification, oocytes successfully fertilized. But, because of their negative electrification, the fertilized egg cannot stick to the uterine wall. As a result, even successfully fertilized oocytes are not attached to the walls of the uterus that leads to the unfortunate result, in general. Thus, the results obtained and the findings allow hoping that the biological value of the information reported by the crystals formed during the evaporation of the female body liquids (Figure 1), in general there is clear.

\section{Conclusion}

The shape of the crystals formed during the evaporation of bodily fluids of the female body, reflects the sign of their electric potential. Changing the electric potential of the female body liquids, you can control over the menstrual cycle, properties of oocytes and fertilization efficiency, in particular artificial. Thus, the onset of the stage of ovulation and efficiency of fertilization can be stimulated by reducing electric potential of biological liquids of the female body.

\section{References}

1. Vasilchenko GS (1990) Sexual pathology. Moscow: Medicine in Russian, Russia.

2. Pivovarenko YV (2016) Nature of the polymorphism of salt crystals in the aspect of arborization diagnostic method. Morphologia 10(1): 72-76.

3. Pivovarenko Y (2017) Potential-Dependent Changes of the Surface Tension of Water. Fluid Mechanics 3(4): 29-32.

4. Nekrasov BV (1974) Bases general chemistry. Moscow: Chemistry in Russian, Russia.

5. Spangenberg JE, Vennemann TW (2008) The stable hydrogen and oxygen isotope variation of water stored in polyethylene terephthalate (PET) bottles. Rapid Commun Mass Spectrom 22: 672-676.

6. Pivovarenko Y (2015) A Charge Distribution in the Earth's Atmosphere. American Journal of Physics and Applications 3(3): 67-68.

7. Pivovarenko Y (2015) UV Absorbance of Aqueous DNA. European Journal of Biophysics 3(3): 19-22.

8. Pivovarenko Y (2017) The Electric Potential of the Tissue Liquids of Living Organisms as a Possible Epigenetic Factor. Chemical and Biomolecular Engineering 2(3): 159-164.

9. Kamzolova SG, Osypov AA, Dzhelyadin TR, Beskaravainy PM, Sorokin AA, et al. (2006) Context-dependent effects of upstream A-tracts on promoter electrostatic properties and function. Proceedings of the Fifth International Conference on Bioinformatics of Genome Regulation and Structure 1: 56-60.

10. Sorokin AA, Osipov AA, Beskaravainyi PM, Kamzolova SG (2007) Analysis of the Distribution of the Nucleotide Sequence and Electrostatic Potential of the Escherichia Coli Genome. Biofizika 52: 223-227.

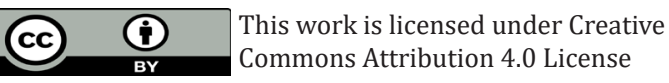

To Submit Your Article Click Here : Submit Article

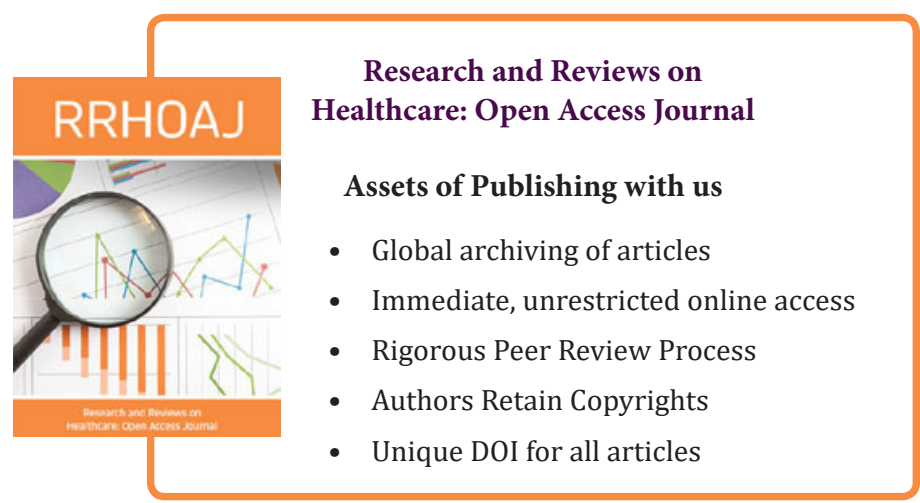

